\title{
Fixed points of nonexpansive potential operators in Hilbert spaces
}

\section{Biagio Ricceri}

"Correspondence: ricceri@dmi.unict.it Department of Mathematics, University of Catania, Viale A Doria 6, Catania, 95125, Italy

\begin{abstract}
In this paper, we show the impact of certain general results by the author on the topic described in the title. Here is a sample:

Let $(X,\langle\cdot, \cdot\rangle)$ be a real Hilbert space and let $T: X \rightarrow X$ be a nonexpansive potential operator.

Then, the following alternative holds: either $T$ has a fixed point, or, for each sphere $S$ centered at 0 , the restriction to $S$ of the functional $x \rightarrow \int_{0}^{1}\langle T(s x), x\rangle d s$ has a unique global maximum towards which each maximizing sequence in $S$ converges.

MSC: 47H09; 47H10; 47J30; 47N10; 49K40; 90C31
\end{abstract}

Keywords: nonexpansive operator; potential operator; fixed point; well-posedness

\section{Introduction}

There is no doubt that fixed point theory for nonexpansive mappings is one of the central topics in modern analysis. Actually, since $[1,4,5]$, such a theory has had (and continues to have) a strong development, and several deep (often spectacular) results have been achieved within it in the settings such as abstract harmonic analysis (where the contributions of Professor Lau are fundamental) and the geometry of Banach spaces.

On the other hand, another very important class of operators is that composed of potential operators. That is to say, the operators that can be regarded as the Gâteaux derivative of a suitable functional. Actually, the variational methods to study linear and nonlinear equations are fully based on potential operators.

In the present paper, as the title says, we are interested in fixed point theory for the intersection of the two above classes of operators in the setting of Hilbert spaces. More precisely, we intend to show the impact of certain general results that the author has established in the last years on such a topic.

Referring to [11] for a thorough introduction to potential operators (with several examples related to them), we recall here a specific situation where one can easily appreciate the relationships between the two classes of operators we are dealing with. Namely, let $(X,\langle\cdot, \cdot\rangle)$ be a real Hilbert space, $L: X \rightarrow X$ a continuous linear operator and $z \in X$. Then, $L+z$ is a potential operator if and only if

$$
\langle L(x), y\rangle=\langle L(y), x\rangle
$$

for all $x, y \in X$, while it is nonexpansive if and only if $\|L\|_{\mathcal{L}(X)} \leq 1$. 
The following result subsumes very well the spirit of the ones that we will establish in Section 3:

Theorem 1.1 Let $(X,\langle\cdot, \cdot\rangle)$ be a real Hilbert space and let $T: X \rightarrow X$ be a nonexpansive potential operator. Then, the following alternative holds: either $T$ has a fixed point, or, for each sphere $S$ centered at 0 , the restriction to $S$ of the functional $x \rightarrow \int_{0}^{1}\langle T(s x), x\rangle d s$ has a unique global maximum towards which each maximizing sequence in $S$ converges.

\section{Preliminaries}

From now on, $(X,\langle\cdot, \cdot\rangle)$ will be a real Hilbert space.

For each $r>0$, we put

$$
\begin{aligned}
& B_{r}=\left\{x \in X:\|x\|^{2}<r\right\}, \\
& \bar{B}_{r}=\left\{x \in X:\|x\|^{2} \leq r\right\}
\end{aligned}
$$

and

$$
S_{r}=\left\{x \in X:\|x\|^{2}=r\right\} .
$$

$T: X \rightarrow X$ will be a nonexpansive operator, i.e.,

$$
\|T(x)-T(y)\| \leq\|x-y\|
$$

for all $x, y \in X$. We also assume that there exists a Gâteaux differentiable functional $J: X \rightarrow \mathbf{R}$, with $J(0)=0$, such that $J^{\prime}=T$, where $J^{\prime}$ is the Gâteaux derivative of $J$. This amounts to say that

$$
\lim _{\lambda \rightarrow 0^{+}} \frac{J(x+\lambda y)-J(x)}{\lambda}=\langle T(x), y\rangle
$$

for all $x, y \in X$. It can easily be checked that

$$
J(x)=\int_{0}^{1}\langle T(s x), x\rangle d s
$$

for all $x \in X$.

We also put

$$
I_{\lambda}(x)=\frac{1}{2}\|x\|^{2}-\lambda J(x)
$$

for all $x \in X, \lambda \in \mathbf{R}$. For $\lambda=1$ we will simply use the symbol $I$ instead of $I_{1}$.

The basic proposition which relates the fixed points of $\lambda T(|\lambda| \leq 1)$ with the functional $I_{\lambda}$ is as follows.

Proposition 2.1 The functional $I_{\lambda}$ is strictly convex and coercive for $|\lambda|<1$, and convex for $|\lambda|=1$. Hence, for each $\lambda \in[-1,1]$, the fixed points of $\lambda T$ agree with the global minima of the functional $I_{\lambda}$. 
Proof Let $|\lambda| \leq 1$. For each $x, y \in X$, we have

$$
\begin{aligned}
\langle x-\lambda T(x)-y+\lambda T(y), x-y\rangle & =\|x-y\|^{2}-\langle\lambda T(x)-\lambda T(y), x-y\rangle \\
& \geq\|x-y\|^{2}-|\lambda|\|T(x)-T(y)\|\|x-y\| \\
& \geq(1-|\lambda|)\|x-y\|^{2} .
\end{aligned}
$$

From this, it follows that the derivative of the functional $I_{\lambda}$ (that is the operator $x \rightarrow$ $x-\lambda T(x))$ is monotone and that it is uniformly monotone if $|\lambda|<1$. Now, the conclusion follows from classical results ([11], pp.247-249).

Another very useful proposition [7] is as follows.

Proposition 2.2 Let $Y$ be a nonempty set, $f, g: Y \rightarrow \mathbf{R}$ two functions, and $\lambda$, $\mu$ two real numbers, with $\lambda<\mu$. Let $\hat{y}_{\lambda}$ be a global minimum of the function $f+\lambda g$ and let $\hat{y}_{\mu}$ be a global minimum of the function $f+\mu g$.

Then, one has

$$
g\left(\hat{y}_{\mu}\right) \leq g\left(\hat{y}_{\lambda}\right)
$$

If either $\hat{y}_{\lambda}$ or $\hat{y}_{\mu}$ is strict and $\hat{y}_{\lambda} \neq \hat{y}_{\mu}$, then

$$
g\left(\hat{y}_{\mu}\right)<g\left(\hat{y}_{\lambda}\right)
$$

Let $S$ be a topological space. As usual, given a function $f: S \rightarrow \mathbf{R}$ and a set $C \subseteq S$, we say that the problem of minimizing (resp. maximizing) $f$ over $C$ is well posed if the following two conditions hold:

- the restriction of $f$ to $C$ has a unique global minimum (resp. maximum), say $\hat{x}$;

- every sequence $\left\{x_{n}\right\}$ in $C$ such that $\lim _{n \rightarrow \infty} f\left(x_{n}\right)=\inf _{C} f$ (resp.

$$
\left.\lim _{n \rightarrow \infty} f\left(x_{n}\right)=\sup _{C} f\right) \text {, converges to } \hat{x} \text {. }
$$

A set of the type $\{x \in S: f(x) \leq r\}$ is said to be a sub-level set of $f$.

Given two functionals $\Phi, \Psi: X \rightarrow \mathbf{R}$, for each $\lambda \in]-\infty,+\infty]$, we denote by $M(\Phi, \Psi, \lambda)$ either the set of all global minima of $\Psi+\lambda \Phi$ or the empty set according to whether $\lambda \in \mathbf{R}$ or $\lambda=+\infty$. We adopt the conventions $\inf \emptyset=+\infty, \sup \emptyset=-\infty$. We also set

$$
\begin{aligned}
& \alpha(\Phi, \Psi, \lambda):=\max \left\{\inf _{X} \Phi, \sup _{M(\Phi, \Psi, \lambda)} \Phi\right\}, \\
& \beta(\Phi, \Psi, \lambda):=\min \left\{\sup _{X} \Phi, \inf _{M(\Phi, \Psi, \lambda)} \Phi\right\} .
\end{aligned}
$$

Note that, by Proposition 2.2, if $a<b$, one has

$$
\alpha(\Phi, \Psi, b) \leq \beta(\Phi, \Psi, a) .
$$

In [8], we established the following basic result:

Theorem 2.1 Let $\Phi, \Psi: X \rightarrow \mathbf{R}$ and let $a, b \in]-\infty,+\infty]$, with $a<b$. Assume that

$$
\alpha(\Phi, \Psi, b)<\beta(\Phi, \Psi, a)
$$


and that, for each $\lambda \in] a, b[$, the functional $\Psi+\lambda \Phi$ has weakly compact sub-level sets and admits a unique global minimum in $X$.

Then, for each $r \in] \alpha(\Phi, \Psi, b), \beta(\Phi, \Psi, a)\left[\right.$, the problem of minimizing $\Psi$ over $\Phi^{-1}(r)$ is well posed with respect to the weak topology. More precisely, the unique global minimum of $\Psi_{\mid \Phi^{-1}(r)}$, say $\hat{x}_{r}$, agrees with the unique global minimum of $\Psi+\lambda \Phi$ for some $\left.\lambda \in\right] a, b[$. Moreover, the functions $r \rightarrow \hat{x}_{r}$ and $r \rightarrow \Psi\left(\hat{x}_{r}\right)$ are continuous in $] \alpha(\Phi, \Psi, a), \beta(\Phi, \Psi, b)[$ with respect to the weak topology.

Finally, let us recall the result of M. Schechter and K. Tintarev [10] that we will apply jointly with Theorem 2.1 in the next section.

Theorem 2.2 Assume that $J$ is sequentially weakly continuous. For each $r>0$, set

$$
\psi(r)=\sup _{x \in S_{r}} J(x)
$$

Moreover, let $A \subseteq] 0,+\infty[$ be an open interval such that, for each $r \in A$,J has no local maxima in $B_{r}$ and there exists a unique $\hat{x}_{r} \in S_{r}$ satisfying $J\left(\hat{x}_{r}\right)=\psi(r)$.

Then, the following conclusions hold:

(i) the function $\psi$ is $C^{1}$ and increasing in $A$;

(ii) for each $r \in A$, one has

$$
T\left(\hat{x}_{r}\right)=2 \psi^{\prime}(r) \hat{x}_{r}
$$

\section{Results}

Our first result (inspired by [6]) shows the key role which a certain function $\varphi:] 0,+\infty[\rightarrow$ $[0,+\infty[$ plays in dealing with the fixed points of $T$.

Theorem 3.1 For each $r>0$, put

$$
\varphi(r)=\inf _{x \in B_{r}} \frac{\sup _{B_{r}} J-J(x)}{r-\|x\|^{2}} .
$$

If there is $r>0$ such that $\varphi(r)<\frac{1}{2}$, then $T$ has a fixed point which lies in $B_{r}$. If $T(0) \neq 0$, then one has

$$
\liminf _{r \rightarrow 0^{+}} \varphi(r) \geq \frac{1}{2}
$$

In any case, one has

$$
\limsup _{r \rightarrow+\infty} \varphi(r) \leq \frac{1}{2}
$$

Proof Let $r>0$ be such that $\varphi(r)<\frac{1}{2}$. So, there exists $x_{0} \in B_{r}$, such that

$$
\frac{\sup _{B_{r}} J-J\left(x_{0}\right)}{r-\left\|x_{0}\right\|^{2}}<\frac{1}{2} .
$$


By Proposition 2.1, $I$ is weakly lower semicontinuous, and so there exists $\hat{x} \in \bar{B}_{r}$ such that

$$
I(\hat{x}) \leq I(x)
$$

for all $x \in \bar{B}_{r}$. We claim that $\|\hat{x}\|^{2}<r$. Actually, if $\|\hat{x}\|^{2}=r$, then, by (3.1), taking into account that $\sup _{B_{r}} J=\sup _{\bar{B}_{r}} J$, we would have

$$
\frac{1}{2}\left\|x_{0}\right\|^{2}-J\left(x_{0}\right)<\frac{1}{2} r-\sup _{y \in \bar{B}_{r}} J(y) \leq \frac{1}{2}\|\hat{x}\|^{2}-J(\hat{x})
$$

against (3.2). As a consequence, $\hat{x}$ is a local minimum of the functional $I$, and so it is a fixed point of $T$, by Proposition 2.1 again.

Now, assume that $T(0) \neq 0$. Arguing by contradiction, suppose that

$$
\liminf _{r \rightarrow 0^{+}} \varphi(r)<\frac{1}{2}
$$

Then, we could find a sequence of positive numbers $\left\{r_{n}\right\}$ converging to 0 such that $\varphi\left(r_{n}\right)<$ $\frac{1}{2}$ for all $n \in \mathbf{N}$. But then, for each $n \in \mathbf{N}$, there would be a fixed point $x_{n}$ of $T$ lying in $B_{r_{n}}$. Hence, $\left\{x_{n}\right\}$ would converge to 0 in $X$ and so, by continuity, we would have $T(0)=0$.

Now, to prove the third assertion, assume that $T$ has a fixed point, say $\tilde{x}$. Then, by Proposition 2.1, we have

$$
\frac{1}{2}\|\tilde{x}\|^{2}-J(\tilde{x}) \leq \frac{1}{2}\|x\|^{2}-J(x)
$$

for all $x \in X$. Fix $r>\|\tilde{x}\|^{2}$. From (3.3), we then obtain

$$
\sup _{B_{r}} J \leq \frac{1}{2}\left(r-\|\tilde{x}\|^{2}\right)+J(\tilde{x})
$$

and so

$$
\varphi(r) \leq \frac{\sup _{B_{r}} J-J(\tilde{x})}{r-\|\tilde{x}\|^{2}} \leq \frac{1}{2}
$$

This clearly implies that

$$
\limsup _{r \rightarrow+\infty} \varphi(r) \leq \frac{1}{2}
$$

Fix $\lambda \in] 0,1[$. So, $\lambda T$ has a (unique) fixed point. By the previous remark, we clearly infer that

$$
\limsup _{r \rightarrow+\infty} \lambda \varphi(r) \leq \frac{1}{2}
$$

and so the conclusion is obtained passing to the limit for $\lambda$ tending to 1 .

Note the following corollary of Theorem 3.1. 
Corollary 3.1 If $T$ has no fixed points, then

$$
\lim _{r \rightarrow+\infty} \varphi(r)=\inf _{r>0} \varphi(r)=\frac{1}{2} \text {. }
$$

The next two results come from Theorem 2.1. Clearly, fix $(T)$ (resp. fix $(-T)$ ) will denote the set of all fixed points of $T$ (resp. $-T$ ).

Theorem 3.2 Assume that $T(0) \neq 0$. Set

$$
\eta_{1}=\max \left\{\inf _{X} J, \sup _{\text {fix }(-T)} J\right\}, \quad \theta_{1}=\min \left\{\sup _{X} J, \inf _{\operatorname{fix}(T)} J\right\} .
$$

For each $\lambda \in]-1,1\left[\right.$, let $\hat{y}_{\lambda}$ be the unique fixed point of the operator $\lambda T$.

Then, the following assertions hold:

$\left(a_{1}\right)$ the function $\lambda \rightarrow g(\lambda):=J\left(\hat{y}_{\lambda}\right)$ is increasing in $]-1,1[$ and its range is $] \eta_{1}, \theta_{1}[$;

$\left(a_{2}\right)$ for each $\left.r \in\right] \eta_{1}, \theta_{1}\left[\right.$, the point $\hat{x}_{r}:=\hat{y}_{g^{-1}(r)}$ is the unique point of minimal norm of $J^{-1}(r)$ towards which every minimizing sequence in $J^{-1}(r)$, for the norm, converges;

(a) the function $r \rightarrow \hat{x}_{r}$ is continuous in $] \eta_{1}, \theta_{1}[$.

Proof We apply Theorem 2.1 taking

$$
\begin{aligned}
& a=-1, \\
& b=1
\end{aligned}
$$

and

$$
\begin{aligned}
& \Phi(x)=-J(x), \\
& \Psi(x)=\frac{1}{2}\|x\|^{2}
\end{aligned}
$$

for all $x \in X$. With these choices, we have

$$
\alpha(\Phi, \Psi, b)=-\theta_{1}
$$

and

$$
\beta(\Phi, \Psi, a)=-\eta_{1}
$$

By Propositions 2.1 and 2.2, the function $g$ is non-decreasing in ]-1,1[ and $g(]-1,1[) \subseteq$ $\left[\eta_{1}, \theta_{1}\right]$. Now, let $\left.A \subset\right]-1,1[$ be a non-degenerate interval. If $g$ was constant in $A$, then, by Proposition 2.2 again, the function $\lambda \rightarrow \hat{y}_{\lambda}$ would be constant in $A$. Let $\hat{y}$ be its unique value. Then, we would have

$$
\hat{y}=\lambda T(\hat{y})
$$

for all $\lambda \in A$. This would imply that $T(\hat{y})=0$, and so $T(0)=0$, against the assumption. Consequently, $g$ is increasing in ]-1,1[. Since, for $|\lambda|<1$, the functional $I_{\lambda}$ is weakly lower 
semicontinuous, coercive and with a unique global minimum (that is $\hat{y}_{\lambda}$ ), we are allowed to apply Theorem 2.1. Accordingly, for each $r \in] \eta_{1}, \theta_{1}\left[\right.$, there exists $\left.\lambda_{r} \in\right]-1,1\left[\right.$, with $\hat{y}_{\lambda_{r}} \in$ $J^{-1}(r)$, such that

$$
\left\|\hat{y}_{\lambda_{r}}\right\|<\|x\|
$$

for all $x \in J^{-1}(r) \backslash\left\{\hat{y}_{\lambda_{r}}\right\}$, and each sequence $\left\{y_{n}\right\}$ in $J^{-1}(r)$ with $\lim _{n \rightarrow \infty}\left\|y_{n}\right\|=\left\|\hat{y}_{\lambda_{r}}\right\|$, weakly converges to $\hat{y}_{\lambda_{r}}$. Since $X$ is a Hilbert space, this implies that $\left\{y_{n}\right\}$ strongly converges to $\hat{y}_{\lambda_{r}}$. Likewise, we get the strong continuity in ] $\eta_{1}, \theta_{1}$ [ of the function $r \rightarrow \hat{y}_{\lambda_{r}}$ from its weak continuity that is ensured by Theorem 2.1 too. Now, to get $\left(a_{1}\right),\left(a_{2}\right),\left(a_{3}\right)$, it is enough to observe that

$$
\lambda_{r}=g^{-1}(r)
$$

for all $r \in] \eta_{1}, \theta_{1}[$.

Theorem 3.3 Assume that $T(0) \neq 0$. Set

$$
\theta_{2}=\inf _{x \in \operatorname{fix}(T)}\|x\|^{2}
$$

For each $\lambda>1$, let $\hat{u}_{\lambda}$ be the unique fixed point of the operator $\frac{1}{\lambda} T$.

Then, the following assertions hold:

(b $\left.b_{1}\right)$ the function $\lambda \rightarrow h(\lambda):=\left\|\hat{u}_{\lambda}\right\|^{2}$ is decreasing in $] 1,+\infty[$ and its range is $] 0, \theta_{2}[$;

$\left(b_{2}\right)$ for each $\left.r \in\right] 0, \theta_{2}\left[\right.$, the point $\hat{v}_{r}:=\hat{u}_{h^{-1}(r)}$ is the unique global maximum of $J_{\mid S_{r}}$ towards which every maximizing sequence for $J_{\mid S_{r}}$ converges;

$\left(b_{3}\right)$ the function $r \rightarrow \hat{v}_{r}$ is continuous in $] 0, \theta_{2}[$.

If, in addition, the functional $J$ is sequentially weakly continuous and has no local maxima in $B_{\theta_{2}}$, then, with $\psi$ defined as in Theorem 2.2, the following further assertions hold:

$\left(b_{4}\right)$ the function $\psi$ is $C^{1}$, increasing and strictly concave in $] 0, \theta_{2}[$;

$\left(b_{5}\right)$ one has

$$
T\left(\hat{v}_{r}\right)=2 \psi^{\prime}(r) \hat{v}_{r}
$$

for all $r \in] 0, \theta_{2}[$;

(b6) one has

$$
\psi^{\prime}(r)=\frac{1}{2} h^{-1}(r)
$$

for all $r \in] 0, \theta_{2}[$.

Proof This time, we apply Theorem 2.1 taking

$$
\begin{aligned}
& a=1, \\
& b=+\infty
\end{aligned}
$$


and

$$
\begin{aligned}
& \Phi(x)=\frac{1}{2}\|x\|^{2}, \\
& \Psi(x)=-J(x)
\end{aligned}
$$

for all $x \in X$. With these choices, we have

$$
\alpha(\Phi, \Psi, b)=0
$$

and

$$
\beta(\Phi, \Psi, a)=\frac{1}{2} \theta_{2}
$$

Since $0 \notin \operatorname{fix}(T)$ and fix $(T)$ is closed, we have $\theta_{2}>0$. Of course, for each $\lambda>1$, the functional $x \rightarrow \frac{\lambda}{2}\|x\|^{2}-J(x)$ is weakly lower semicontinuous, coercive and with a unique global minimum (that is $\hat{u}_{\lambda}$ ), and so we can derive $\left(b_{1}\right),\left(b_{2}\right),\left(b_{3}\right)$ from Theorem 2.1, reasoning as in the proof of Theorem 3.2. Under the additional assumptions on $J,\left(b_{4}\right),\left(b_{5}\right)$ follow directly from Theorem 2.2, taking $A=] 0, \theta_{2}$ [. Finally, $\left(b_{6}\right)$ is a consequence of $\left(b_{5}\right)$ and of the fact that $T\left(\hat{v}_{r}\right)=h^{-1}(r) \hat{v}_{r}$.

Remark 3.1 Of course, Theorem 1.1 is a by-product of Theorem 3.3, as, if $T$ has no fixed points, we have $\theta_{2}=+\infty$. On the other hand, if, for some $r>0$, the problem of maximizing $J$ over $S_{r}$ is not well posed, then $T$ has a fixed point lying in $\bar{B}_{r}$. Indeed, from $\left(b_{2}\right)$ it follows that $r \geq \theta_{2}$. But, fix $(T)$ is a closed and convex set. So, it admits a point of minimal norm. By the above inequality, such a point lies in $\bar{B}_{r}$ and we are done.

Now, we want to present the form that Theorem 3.3 assumes when $T$ is an affine operator.

As usual, for a linear operator $L: X \rightarrow X$, we say that

- $L$ is compact if, for each bounded set $C \subset X$, the set $\overline{L(C)}$ is compact;

- $L$ is symmetric if

$$
\langle L(x), u\rangle=\langle L(u), x\rangle
$$

for all $x, u \in X$.

Theorem 3.4 Let $L: X \rightarrow X$ be a symmetric continuous linear operator, with norm 1 , and let $z \in X \backslash\{0\}$.

For each $\lambda>1$, let $\hat{w}_{\lambda}$ be the unique fixed point of the operator $\frac{1}{\lambda}(L-z)$. Moreover, set

$$
\theta=\inf _{x \in \operatorname{fix}(L-z)}\|x\|^{2}
$$

and, for each $r>0$,

$$
\delta(r)=\sup _{x \in S_{r}} H(x)
$$


where

$$
H(x)=\left\langle\frac{1}{2} L(x)-z, x\right\rangle .
$$

Then, the following assertions hold:

$\left(c_{1}\right)$ the function $\lambda \rightarrow k(\lambda):=\left\|w_{\lambda}\right\|^{2}$ is decreasing in $] 1,+\infty[$ and its range is $] 0, \theta[$;

$\left(c_{2}\right)$ for each $\left.r \in\right] 0, \theta\left[\right.$, the point $\hat{\omega}_{r}:=\hat{w}_{k^{-1}(r)}$ is the unique global maximum of $H_{\mid S_{r}}$ towards which every maximizing sequence for $H_{\mid S_{r}}$ converges;

$\left(c_{3}\right)$ the function $r \rightarrow \hat{\omega}_{r}$ is continuous in $] 0, \theta[$.

If, in addition, $T$ is compact, then the following further assertions hold:

$\left(c_{4}\right)$ the function $\delta$ is $C^{1}$, increasing and strictly concave in $] 0, \theta[$;

$\left(c_{5}\right)$ one has

$$
L\left(\hat{\omega}_{r}\right)-2 \delta^{\prime}(r) \hat{\omega}_{r}=z
$$

for all $r \in] 0, \theta[$

$\left(c_{6}\right)$ one has

$$
\delta^{\prime}(r)=\frac{1}{2} k^{-1}(r)
$$

for all $r \in] 0, \theta[$.

Before giving the proof of Theorem 3.4, we establish the following

Proposition 3.1 Let $L: X \rightarrow X$ be a symmetric continuous linear operator and let $H$ be defined as in Theorem 3.4.

Then, for $\tilde{x} \in X$, the following are equivalent:

(j) $\tilde{x}$ is a local maximum of $H$.

(jj) $\tilde{x}$ is a global maximum of $H$.

(jij) $L(\tilde{x})=z$ and $\sup _{x \in X}\langle L(x), x\rangle \leq 0$.

Proof First, observe that the symmetry of $L$ is equivalent to the fact that the functional $H$ is Gâteaux differentiable with derivative given by

$$
H^{\prime}(x)=L(x)-z
$$

for all $x \in X$ ([11], p.235). By the symmetry of $L$ again, it is easy to check that, for each $x \in X$, the inequality

$$
H(\tilde{x}+x) \leq H(\tilde{x})
$$

is equivalent to

$$
\left\langle L(\tilde{x})-z+\frac{1}{2} L(x), x\right\rangle \leq 0 .
$$


Now, if (j) holds, then $H^{\prime}(\tilde{x})=0$ (that is $L(\tilde{x})=z$ ) and there is $r>0$ such that (3.4) holds for all $x \in B_{r}$. So, from (3.5), we have $\langle L(x), x\rangle \leq 0$ for all $x \in B_{r}$ and then, by linearity, for all $x \in X$, and this shows (jji). Vice versa, if (jji) holds, then (3.5) is satisfied for all $x \in X$ and so by (3.4), $\tilde{x}$ is a global maximum of $H$, and the proof is complete.

Proof of Theorem 3.4 As we observed above, the symmetry of $L$ is equivalent to the fact that $L$ agrees with the derivative of $H$. So, since $z \neq 0$, we can apply Theorem 3.3 taking $T=L-z$. In such a way, we derive $\left(c_{1}\right)-\left(c_{3}\right)$ directly from $\left(b_{1}\right)-\left(b_{3}\right)$. Now, assume that $L$ is also compact. Then, this implies that $H$ is sequentially weakly continuous ([11], Corollary 41.9). Suppose that $H$ has a local maximum, say $\tilde{x}$. Then, by Proposition 3.1, $\tilde{x}$ is a global maximum of $H$. In particular, this implies that the functional $x \rightarrow \frac{1}{2}\|x\|^{2}-H(x)$ is coercive and hence, by sequential weak lower semicontinuity, it has a global minimum. That is, fix $(L-z) \neq \emptyset$, by Proposition 2.1. So, by Proposition 2.2, it clearly follows that

$$
\|\tilde{x}\|^{2} \geq \theta
$$

In other words, $H$ has no local maxima in $B_{\theta}$. At this point, $\left(c_{4}\right)-\left(c_{6}\right)$ come directly from $\left(b_{4}\right)-\left(b_{6}\right)$.

Some remarks on Theorem 3.4 are now in order.

Remark 3.2 Note that the compactness of $L$ serves only to guarantee that the functional $x \rightarrow\langle L(x), x\rangle$ is sequentially weakly continuous. So, Theorem 3.4 actually holds under such a weaker condition.

Remark 3.3 A natural question is: if assertions $\left(c_{1}\right)-\left(c_{6}\right)$ hold, must the operator $L$ be symmetric and the functional $x \rightarrow\langle L(x), x\rangle$ sequentially weakly continuous?

Remark 3.4 Note that if $L$, besides being compact and symmetric, is also positive (i.e., $\inf _{x \in X}\langle L(x), x\rangle \geq 0$ ), then, by classical results, the operator $x \rightarrow L(x)-x$ is not surjective, and so there are $z \in X$ for which the conclusion of Theorem 3.4 holds with $\theta=+\infty$.

In the previous results, the essential assumption is that $T(0) \neq 0$. In the next (and last) result, to the contrary, we highlight a remarkable uniqueness property occurring when $\sup _{X} J=0$ (and so $T(0)=0$ ). Actually, in such a case, 0 is the unique fixed point of $\lambda T$ for each $\lambda \in] 0,3[$.

More precisely, for each real Hilbert space $(Y,\langle\cdot, \cdot\rangle)$, we denote by $\mathcal{A}_{Y}$ the class of all nonexpansive potential operators $P: Y \rightarrow Y$ such that

$$
\sup _{x \in Y} \int_{0}^{1}\langle P(s x), x\rangle d s=0 .
$$

Set

$$
\gamma_{Y}=\inf _{P \in \mathcal{A}_{Y}} \inf \{\lambda>0: x=\lambda P(x) \text { for some } x \neq 0\}
$$

We have: 
Theorem 3.5 For any real Hilbert space $(Y,\langle\cdot, \cdot\rangle)$, with $Y \neq\{0\}$, one has

$\gamma_{Y}=3$

We first prove

Proposition 3.2 One has

$\gamma_{\mathbf{R}}=3$.

Proof Let $P \in \mathcal{A}_{\mathbf{R}}$. Fix $\left.\lambda \in\right] 0,3[$. Let us prove that 0 is the only fixed point of $\lambda P$. Arguing by contradiction, assume that $x_{0}$ is a non-zero fixed point of $\lambda P$. It is not restrictive to assume that $x_{0}>0$ (otherwise, we would work with $P(-x)$ ). Consider now the function $\chi: \mathbf{R} \rightarrow \mathbf{R}$ defined by

$$
\chi(x)= \begin{cases}-\frac{x^{2}}{2} & \text { if } x<\frac{x_{0}}{3} \\ \frac{x^{2}}{2}-\frac{2 x_{0} x}{3}+\frac{x_{0}^{2}}{9} & \text { if } \frac{x_{0}}{3} \leq x \leq x_{0} \\ -\frac{x^{2}}{2}+\frac{4 x_{0} x}{3}-\frac{8 x_{0}^{2}}{9} & \text { if } x_{0}>x\end{cases}
$$

Clearly, $\chi \in C^{1}(\mathbf{R})$. Let $x>0$. If $0<x \leq \frac{x_{0}}{3}$, we have

$$
\chi^{\prime}(x)=-x \leq P(x)
$$

If $x>\frac{x_{0}}{3}$, we have

$$
\chi^{\prime}(x)=\frac{x_{0}}{3}-\left|x-x_{0}\right|=\frac{\lambda P\left(x_{0}\right)}{3}-\left|x-x_{0}\right|<P\left(x_{0}\right)-\left|x-x_{0}\right| \leq P(x) .
$$

So, in particular, we get

$$
\frac{4 x_{0}}{3} \int_{0}^{1} P\left(\frac{4 x_{0}}{3} s\right) d s=\int_{0}^{\frac{4 x_{0}}{3}} P(x) d x>\int_{0}^{\frac{4 x_{0}}{3}} \chi^{\prime}(x) d x=\chi\left(\frac{4 x_{0}}{3}\right)=0
$$

which contradicts the fact $P \in \mathcal{A}_{\mathbf{R}}$. From what we have just proven, it clearly follows that

$$
3 \leq \gamma_{\mathbf{R}}
$$

Now, fix any $\mu>1$. Continue to consider the function $\chi$ defined above (for a fixed $x_{0}>0$ ). Clearly, the function $\frac{1}{\mu} \chi^{\prime}$ belongs to $\mathcal{A}_{\mathbf{R}}$ and

$$
x_{0}=3 \mu \frac{\chi^{\prime}\left(x_{0}\right)}{\mu} .
$$

Of course, from this we infer that

$$
\gamma_{\mathbf{R}} \leq 3 \mu,
$$

and the conclusion clearly follows. 
Proof of Theorem 3.5 First, let us prove that

$$
\gamma_{Y} \leq 3
$$

To this end, fix any $Q \in \mathcal{A}_{\mathbf{R}}$ and any $\lambda>0$ such that

$$
\hat{t}=\lambda Q(\hat{t})
$$

for some $\hat{t} \neq 0$. Fix also $u \in Y$, with $\|u\|=1$, and consider the operator $P: Y \rightarrow Y$ defined by

$$
P(x)=Q(\langle u, x\rangle) u
$$

for all $x \in Y$. Clearly, $P \in \mathcal{A}_{Y}$. Finally, set

$$
\hat{x}=\lambda Q(\hat{t}) u \text {. }
$$

Of course, $\hat{x} \neq 0$. Since

$$
\langle u, \hat{x}\rangle=\lambda Q(\hat{t})
$$

we also have

$$
\langle u, \hat{x}\rangle=\hat{t}
$$

and so

$$
\hat{x}=\lambda P(\hat{x}) .
$$

From this, it clearly follows that

$$
\gamma_{Y} \leq \gamma_{\mathbf{R}}
$$

and so (3.6) follows now from Proposition 3.2.

Now, let us prove that

$$
3 \leq \gamma_{Y} .
$$

To this end, fix $P \in \mathcal{A}_{Y}, \lambda>0$ and $\tilde{x} \in Y \backslash\{0\}$ such that

$$
\tilde{x}=\lambda P(\tilde{x}) .
$$

Then, consider the function $Q: \mathbf{R} \rightarrow \mathbf{R}$ defined by

$$
Q(t)=\left\langle P\left(\frac{t \tilde{x}}{\|\tilde{x}\|}\right), \frac{\tilde{x}}{\|\tilde{x}\|}\right\rangle
$$


for all $t \in \mathbf{R}$. Clearly, the primitive of $Q$ vanishing at 0 is non-positive in $\mathbf{R}$. Moreover, for each $t, s \in \mathbf{R}$, we have

$$
\begin{aligned}
|Q(t)-Q(s)| & =\left|\left\langle P\left(\frac{t x}{\|x\|}\right)-P\left(\frac{s x}{\|x\|}\right), \frac{x}{\|x\|}\right\rangle\right| \\
& \leq\left\|P\left(\frac{t x}{\|x\|}\right)-P\left(\frac{s x}{\|x\|}\right)\right\| \leq|t-s| .
\end{aligned}
$$

This shows that $Q$ is nonexpansive, and so $Q \in \mathcal{A}_{\mathbf{R}}$. Now, from (3.8), we get

$$
\|\tilde{x}\|=\lambda\left\langle P(\tilde{x}), \frac{\tilde{x}}{\|\tilde{x}\|}\right\rangle
$$

that is

$$
\|\tilde{x}\|=\lambda Q(\|\tilde{x}\|)
$$

From this, we infer that

$$
\gamma_{\mathbf{R}} \leq \gamma_{Y}
$$

So (3.7) follows from Proposition 3.2, and the proof is complete.

For specific consequences of Theorem 3.5 concerning nonlinear elliptic equations, we refer to the very interesting papers [2] and [3] where a problem asked in [9] was solved.

\section{Competing interests}

The author declares that he has no competing interest.

\section{Acknowledgement}

Dedicated to Professor Anthony To-Ming Lau, with esteem and friendship.

Received: 4 April 2012 Accepted: 10 July 2012 Published: 24 July 2012

\section{References}

1. Browder, FE: Nonexpansive nonlinear operators in a Banach space. Proc. Natl. Acad. Sci. USA 54, 1041-1044 (1965)

2. Fan, $\mathrm{XL}$ : A remark on Ricceri's conjecture for a class of nonlinear eigenvalue problems. J. Math. Anal. Appl. 349, 436-442 (2009)

3. Fan, XL: On Ricceri's conjecture for a class of nonlinear eigenvalue problems. Appl. Math. Lett. 22, 1386-1389 (2009)

4. Göhde, D: Zum Prinzip der kontraktiven Abbildung. Math. Nachr. 30, 251-258 (1965)

5. Kirk, WA: A fixed point theorem for mappings which do not increase distances. Am. Math. Mon. 72, 1004-1006 (1965)

6. Ricceri, B: A general variational principle and some of its applications. J. Comput. Appl. Math. 113, 401-410 (2000)

7. Ricceri, B: Uniqueness properties of functionals with Lipschitzian derivative. Port. Math. 63, 393-400 (2006)

8. Ricceri, B: Well-posedness of constrained minimization problems via saddle-points. J. Glob. Optim. 40, 389-397 (2008)

9. Ricceri, B: A remark on a class of nonlinear eigenvalue problems. Nonlinear Anal. 69, 2964-2967 (2008)

10. Schechter, M, Tintarev, K: Spherical maxima in Hilbert space and semilinear elliptic eigenvalue problems. Differ. Integral Equ. 3, 889-899 (1990)

11. Zeidler, E: Nonlinear Functional Analysis and Its Applications, vol. III. Springer, Berlin (1985) 